\title{
New-onset hyperglycemia: a potential clue to detect early pancreatic cancer
}

\section{ABSTRACT}

Pancreatic adenocarcinoma has an incidence rate nearly equal to the mortality rate and this is mostly due to late onset of symptoms and delay in diagnosis. Early diagnosis of this cancer gives the opportunity for total resection of pancreas and creates hope for a full recovery. Compelling evidence now indicates that new-onset diabetes may be a manifestation of occult pancreatic carcinoma. Authors report a young female who presented with new-onset severe hyperglycemia and superficial thrombophlebitis. She was subsequently diagnosed with pancreatic cancer confirmed by histopathology. Her glycemic status evaluated 6 months prior to her presentation during institutional health check-up was entirely normal. This case report will serve to emphasize that new-onset diabetes in certain patients could be a presenting feature of pancreatic cancer. (Clin Diabetol 2017; 6, 3: 115-117)

Key words: pancreatic carcinoma, new-onset hyperglycemia, warning sign, asymptomatic stage

\section{Introduction}

Pancreatic cancer $(\mathrm{PaC})$ carries a poor prognosis since cancer-specific symptoms occur only at an advanced stage. $\mathrm{PaC}$ is associated with a diabetogenic state and evidence has emerged that new-onset diabetes mellitus (DM) may be a warning sign necessitating further investigation for occult PaC [1, 2]. Improvement

Address for correspondence:

Dr. Sayantan Ray

Institute of Post Graduate Medical Education \& Research

244, AJC Bose Road, 700020, Kolkata, India

e-mail: sayantan.ray30@gmail.com

Clinical Diabetology 2017, 6, 3, 115-117

DOI: $10.5603 /$ DK.2017.0019

Received: 16.05.2017

Accepted: 23.07.2017 of diabetes following resection of $\mathrm{PaC}$ suggests that diabetes is caused by the cancer. Patients with new-onset DM have a 5-8-fold increased risk of being diagnosed with $\mathrm{PaC}$ within 1-3 years of developing diabetes [3]. Although the association between new-onset DM and $\mathrm{PaC}$ is established, the pathogenesis of $\mathrm{PaC}$-associated $\mathrm{DM}$ is not well understood. It has been postulated that $\mathrm{PaC}$-associated DM is a paraneoplastic phenomenon caused by diabetogenic tumor-secreted products [4]. Recognition of new-onset hyperglycemia as an early manifestation of $\mathrm{PaC}$ could lead to diagnosis of $\mathrm{PaC}$ at an early asymptomatic stage. The learning objective of the following report is to highlight that new-onset DM in the setting of suspicion or risk for $\mathrm{PaC}$ may serve as a clue that the patient needs further workup for this malignancy.

\section{Case study}

A 32-year-old female with no significant past medical history presented to us with new onset osmotic symptoms and severe hyperglycemia. She underwent a routine health check-up outside about 6 months prior to her presentation and plasma glucose measured during that time was normal. She used to lead a sedentary lifestyle. She had been experiencing polydipsia and polyuria for preceding 3 weeks associated with blurry vision and distal extremity paresthesias. She lost about $7 \mathrm{~kg}$ weight over the last 6 months. Her family history was negative for diabetes and or any cancers. Clinical examination revealed normal body mass index (BMI) $\left(21.5 \mathrm{~kg} / \mathrm{m}^{2}\right)$ and absence of acanthosis. Interestingly, one of the superficial veins of left upper limb was firm and tender on palpation suggestive of thrombophlebitis (Fig. 1). Systemic examination was unremarkable.

Laboratory findings on admission were as follows: random blood glucose $408 \mathrm{mg} / \mathrm{dL}$, white blood cell count 9,500/ $\mu \mathrm{l}$, bicarbonate $23 \mathrm{mmol} / \mathrm{L}$, anion gap 10, and negative serum and urine ketones. Her glycated 
hemoglobin was $11.7 \%$. Ultrasound of abdomen revealed a heterogeneous mass in the body of pancreas. The patient subsequently underwent a CT scan of the abdomen with IV contrast that showed a mass $(3.2 \times$ $3.1 \times 3.3 \mathrm{~cm}$ ) extending from the body into the tail of the pancreas (Fig. 2). Subsequent laboratory studies were significant for an elevated CA 19-9 (349.9 U/mL), normal CA-125 (15 U/mL), and a low post-meal C-peptide $(0.2 \mathrm{ng} / \mathrm{mL})$. CT guided fine needle aspiration of the lesion was performed and was suggestive of pancreatic adenocarcinoma.

Her diabetes was treated with a multiple daily injection (MDI) of insulin glargine (40 units once daily) and insulin aspart 10-20 units three times a day before a meal. After discussion with the patient and her family, we decided to proceed for surgical resection. However, the surgeons found it very difficult to separate the mass from the surrounding structures and the mass was partly resected and a lateral pancreatico-jejunostomy was performed. Histopathology was suggestive of pancreatic adenocarcinoma (Fig. 3). The daily insulin requirement significantly reduced in the immediate post-operative period; however, the patient had a very stormy post-operative course which was complicated by severe sepsis, respiratory distress syndrome and multi-organ failure. Despite our best effort she succumbed to death on $14^{\text {th }}$ post-operative day.

\section{Discussion}

In our patient, new-onset hyperglycemia was a harbinger of undetected pancreatic adenocarcinoma. Patients with $\mathrm{PaC}$ often present with new-onset DM which resolves following cancer resection [4]. Unfortunately, in our case a fatal outcome impeded further monitoring of glycemic status. Largely, $\mathrm{PaC}$-associated diabetes is of recent onset, beginning up to 2 years prior the diagnosis of cancer [1]. Weight loss often manifests before the development of diabetes. Patel et al. reported a middle-aged woman who presented with hyperglycemia and was subsequently diagnosed with new-onset diabetes and biopsy proven metastatic $\mathrm{PaC}$ [5]. $\mathrm{PaC}$ has a poor prognosis as diagnosis is usually made at advanced stages of disease due to delayed symptom onset. To substantially impact long-term survival, early detection of $\mathrm{PaC}$ is needed. Evidence from studies investigating the association between diabetes and $\mathrm{PaC}$ suggests that while long-standing diabetes is a cause for pancreatic cancer, new-onset diabetes is its manifestation $[1,6]$. Considering the modest association between type $2 \mathrm{DM}$ and $\mathrm{PaC}$, the relatively low incidence of $\mathrm{PaC}$, and the fact that type $2 \mathrm{DM}$ is rather prevalent in the general population, screening all subjects with long-standing diabetes for this cancer

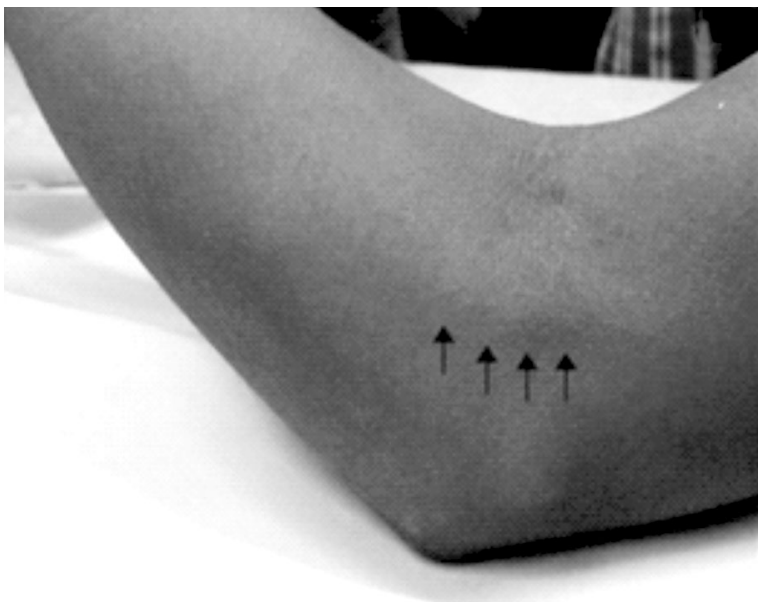

Figure 1. Superficial thrombophlebitis of one of the upper extremity veins (black arrows)

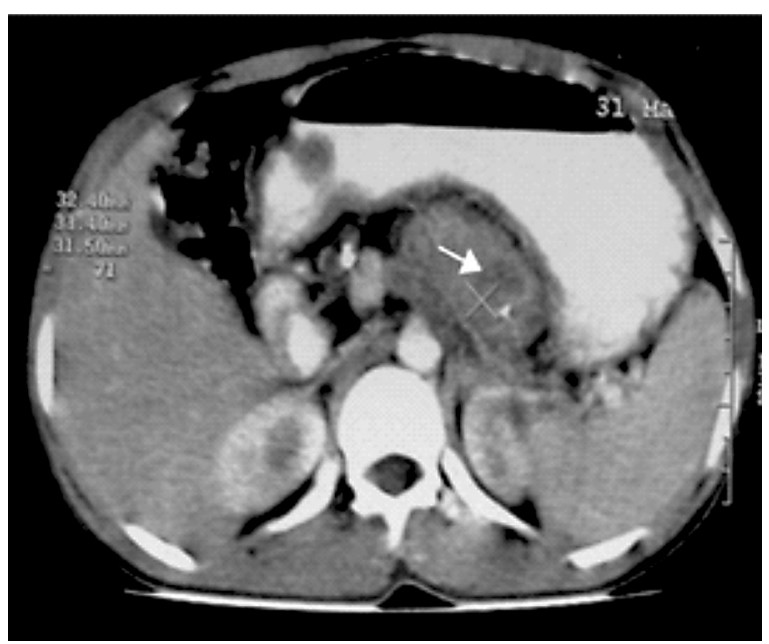

Figure 2. Abdominal CT showing a hetergenously hypodense mildly enhancing SOL $(3.2 \times 3.1 \times 3.3 \mathrm{~cm})$ in the body and tail region of pancreas. Pancreatic anatomy is grossly disorganized and head and neck are not clearly visualized

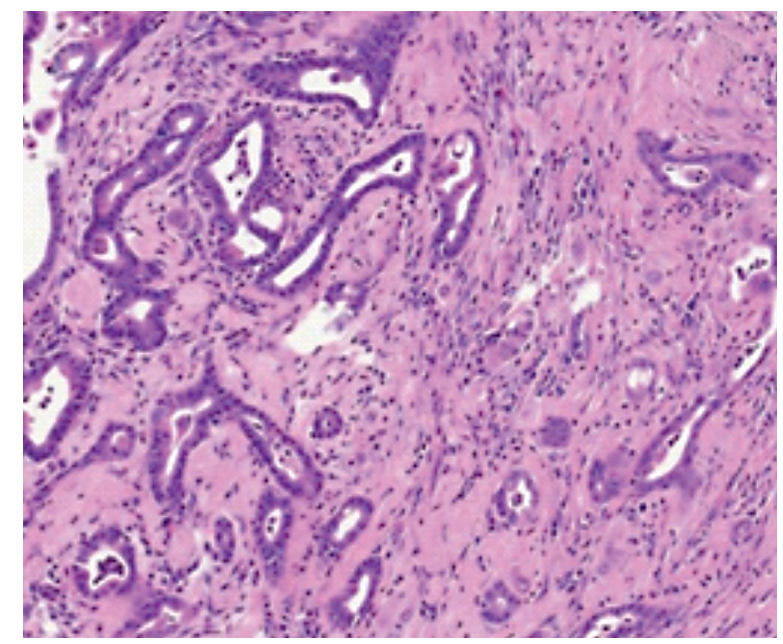

Figure 3. Photomicrograph showing malignant irregular glands haphazardly infiltrating into desmoplastic stroma ( $\mathrm{H}$ and $\mathrm{E}, \times 40$ ). 
will not be cost-effective. Nevertheless, new-onset DM secondary to the cancer appears to be a clinically useful sign of occult PaC [1]. New-onset diabetes is found in almost $50 \%$ of all $\mathrm{PaC}$ and several lines of evidence suggest that diabetes is attributable to the cancer [7, 8]. Recent studies have shown that $\mathrm{PaC}$ detected at the onset of diabetes is usually resectable [8]. Patient with newly diagnosed diabetes should have an ultrasound of the abdomen. In selected cases where there is clinical suspicion or risk factor of $\mathrm{PaC}$ further evaluation should be made with assessment of tumor markers like CA-19-9 and CEA. Both of these markers have shown a positive correlation with $\mathrm{PaC}$-associated diabetes [9].

As in type $2 \mathrm{DM}, \beta$-cell dysfunction and peripheral insulin resistance are seen in $\mathrm{PaC}$-induced diabetes. A low C-peptide level, observed in our case, may indicate a possible coexistence of the factors contributing to insulin resistance and decreased insulin secretion (inhibitory factors produced by cancer or cancer-dependent immune process). Clinical and laboratory evidence supports the hypothesis that PaC-associated DM is a paraneoplastic phenomenon but its biochemical mediator(s) are not well-known [4]. One such possible biomarker is adrenomedullin, which is a potential mediator of $\beta$-cell dysfunction in PaC-associated DM [10]. New-onset DM provides a high-risk group that could be screened for $\mathrm{PaC}$ at a time when the cancer is asymptomatic. However, the success of the strategy to use new-onset $\mathrm{DM}$ as a marker of asymptomatic cancer will depend the ability to distinguish $\mathrm{PaC}$ - associated DM from the more common type $2 \mathrm{DM}$. A clinical distinction between $\mathrm{PaC}$-associated diabetes and type 2DM is sometimes difficult. Future studies should focus on unraveling the pathogenesis and identifying candidate mediator(s) of $\mathrm{PaC}$-associated DM to develop discriminating biomarkers that can identify PaC-associated DM among subjects with new-onset DM.

To conclude, new-onset hyperglycemia has been recognized as a marker of early, asymptomatic $\mathrm{PaC}$.
In future, new-onset hyperglycemia or diabetes could be a potential first sieve for screening of $\mathrm{PaC}$.

\section{Conflict of interest}

The authors have nothing to disclose.

\section{REFERENCES}

1. Pannala R, Basu A, Petersen GM, et al. New-onset diabetes: a potential clue to the early diagnosis of pancreatic cancer. Lancet Oncol. 2009; 10(1): 88-95, doi: 10.1016/S1470-2045(08)70337-1, indexed in Pubmed: 19111249

2. Gupta S, Vittinghoff E, Bertenthal D, et al. New-onset diabetes and pancreatic cancer. Clin Gastroenterol Hepatol. 2006; 4(11): 1366-72; quiz 1301, doi: 10.1016/j.cgh.2006.06.024, indexed in Pubmed: 16945591.

3. Sah RP, Nagpal SJ, Mukhopadhyay D, et al. New insights into pancreatic cancer-induced paraneoplastic diabetes. Nat Rev Gastroenterol Hepatol. 2013; 10(7): 423-433, doi: 10.1038/ /nrgastro.2013.49, indexed in Pubmed: 23528347.

4. Pannala R, Leirness JB, Bamlet WR, et al. Prevalence and clinical profile of pancreatic cancer-associated diabetes mellitus. Gastroenterology. 2008; 134(4): 981-987, doi: 10.1053/j.gastro.2008.01.039, indexed in Pubmed: 18395079.

5. Patel R, Ede J, Collins J, et al. Pancreatic cancer presenting as new-onset diabetes. Case Rep Oncol. 2014; 7(1): 171-174, doi: 10.1159/000360812, indexed in Pubmed: 24748867.

6. Everhart J, Wright D. Diabetes mellitus as a risk factor for pancreatic cancer. A meta-analysis. JAMA. 1995; 273(20): 1605-1609, indexed in Pubmed: 7745774.

7. Permert J, Ihse I, Jorfeldt $L$, et al. Pancreatic cancer is associated with impaired glucose metabolism. Eur J Surg. 1993; 159(2): 101-107, indexed in Pubmed: 8098623.

8. Gangi S, Fletcher JG, Nathan MA, et al. Time interval between abnormalities seen on $\mathrm{CT}$ and the clinical diagnosis of pancreatic cancer: retrospective review of CT scans obtained before diagnosis. AJR Am J Roentgenol. 2004; 182(4): 897-903, doi: 10.2214/ /ajr.182.4.1820897, indexed in Pubmed: 15039161.

9. Guo Q, Kang M, Zhang Bo, et al. Elevated levels of CA 19-9 and CEA in pancreatic cancer-associated diabetes. J Cancer Res Clin Oncol. 2010; 136(11): 1627-1631, doi: 10.1007/s00432-0100820-0, indexed in Pubmed: 20174821.

10. Aggarwal G, Ramachandran V, Javeed N, et al. Adrenomedullin is up-regulated in patients with pancreatic cancer and causes insulin resistance in $\beta$ cells and mice. Gastroenterology. 2012; 143(6): 1510-1517.e1, doi: 10.1053/j.gastro.2012.08.044, indexed in Pubmed: 22960655. 
